L'impensable exil. Réflexion sur Je fais comme fait le nageur dans la mer de Sadek Aissat

\title{
Belaïd Djefel
}

\section{OpenEdition}

\section{Journals}

Édition électronique

URL : http://journals.openedition.org/recherchestravaux/406

DOI : 10.4000/recherchestravaux.406

ISSN : 1969-6434

Éditeur

UGA Éditions/Université Grenoble Alpes

Édition imprimée

Date de publication : 30 juillet 2010

Pagination : 55-64

ISBN : 978-2-84310-174-8

ISSN : 0151-1874

Référence électronique

Belaïd Djefel, «L'impensable exil. Réflexion sur Je fais comme fait le nageur dans la mer de

Sadek Aissat », Recherches \& Travaux [En ligne], 76 | 2010, mis en ligne le 30 janvier 2012, consulté le 08 septembre 2020. URL : http://journals.openedition.org/recherchestravaux/406 ; DOI : https:// doi.org/10.4000/recherchestravaux.406 


\section{L'impensable exil \\ Réflexion sur Je fais comme fait le nageur dans la mer de Sadek Aissat ${ }^{\mathrm{I}}$}

«Émerge autant que possible à ta propre surface. Que le risque soit ta clarté. Comme un vieux rire. Dans une entière modestie.»

René Char

"Ceux qui campent chaque jour plus loin du lieu de leur naissance, ceux qui tirent chaque jour leur barque sur d'autres rives, savent mieux chaque jour le cours des choses illisibles; et remontant les fleuves vers leur source, entre les vertes apparences, ils sont gagnés soudain de cet éclat sévère où toute langue perd ses amarres.»

Saint-John Perse

Le souvenir du lieu gouverne dans toute sa radicalité le texte de Sadek Aissat. C'est, en effet, autour du lieu perdu, symbolisé par les deux lettres évocatrices "D. Z.», que le narrateur, répondant aux mêmes initiales, reconstruit sa mémoire pour tenter de retrouver, dans l'espace exigu d'un "foyer», le rire immense à jamais éteint :

Je me souviens du jour où j'ai entamé ma traversée, quand j'ai eu à affronter mes peurs, et la toute première fois, à m'arracher du rire des miens. Il avait perdu toute fraternité, leur rire, et, ne pouvant me résigner à faire bon commerce de cette déconvenue, ravalant ma rage et changeant mes jours d'épaule, j’ai quitté le navire. (p. 6o)

I. Éditions Barzakh, Alger, 2002. Les références sont données entre parenthèses après les citations. 
Cet acte fondateur, signe d'une grande rupture et véritable tournant, déterminera en totalité l'itinéraire de D. Z., à la recherche de ceux qui, comme lui, sont "pris dans la tourmente des destins qui les dépassent» (p. I46).

Les «miens», ayant désormais perdu la valeur affective, c'est le visage de Sien qui finira par incarner toute la charge symbolique d'une fraternité effective et agissante :

Je ne savais pas, dit d'elle, D. Z., si elle était Arabe ou Française, elle ne m’avait toujours rien dit. Elle avait quelque chose d'arabe avec quelque chose d'asiatique, on eut cru discerner la lueur lointaine d'une généalogie perdue dans un autre âge. (p. 42)

Visage sans visage, ou plutôt, visage qui annule tous les visages, toute marque distinctive, toute origine, Sien est la négation même de toute filiation généalogique attestée. Détail important : tous les personnages du roman échappent aux marquages identitaires traditionnels, ce qui les soustrait, de fait, à la tyrannie de l'origine. Leurs identités respectives, en déjouant les signes totémiques modernes ${ }^{2}$, deviennent les signes fédérateurs de la communauté des sans-patrie, ceux qui «habitent leur hémoptysie et l'intérieur schizophrénique des étrangers qu'ils sont devenus.» (p. 193)

Ainsi est-il préférable de renoncer, pour mieux intégrer l'autre, à toute ambition généalogique. L'identification des personnages se réduit, en effet, au seul prénom, et plus radicalement encore, à des initiales, élargissant ainsi le paradigme des désignations. Ce brouillage de repères réduit à notre sens la distance qui pourrait naître de l'exacerbation identitaire. Avoir un nom et une origine dans un monde sans unité nous empêche de séjourner auprès de tous ceux qui sont "pris dans la tourmente de destins qui les dépassent" (p. I46), et que le désordre du monde a jetés dans l'errance. C'est, paradoxalement, dans l'absence de tout ce qui offre une sécurité et un abri qu’a lieu et se formule nécessairement le questionnement originaire qui fait surgir l'être en son «il est». Les distances qui nous rapprochent de l'autre (ou qui nous en éloignent) peuvent être évaluées à travers le reflet qu'autrui projette sur nous :

Une fille, jeune, [...] était affalée sur la banquette du fond. Assis sur le plancher, entre ses cuisses, la tête reposant sur son bas-ventre, un garçon du même âge. [...]. Ils ne bougeaient pas, paumés, perdus aux frontières acidulées de leurs songes exténués. [...] Ces deux-là paraissaient au bord de la mort. [...]. À les observer, je me sentais moi aussi à la périphérie d'une mort familière, et seul le lieu de la mort me paraissait avoir encore une quelconque importance. Un violent sentiment de compassion, de pitié m’avait submergé et j'avais eu envie de

2. L'expression «pays de personne" brouille les repères identificatoires raciaux et nationalitaires. 
pleurer. Quelque chose de moi, me semblait-il, se réfractait dans l'optique cassée de ces deux enfants, mourants presque, désespérés. (p. 22-23)

C'est la profondeur et la singularité de l'être qu'il convient de réhabiliter : cet être désintégré que des années de vie d'exploitation, dans la grande société de consommation pourvoyeuse d'anonymat, ont enfoui, maquillé et dénaturé au point de le rendre sinon inconnaissable du moins malaisément connaissable. C'est ainsi que sont fabriqués les "gens anormaux", les "apatrides " qui rusent et passent leur temps à

[...] déjouer la vigilance du gardien des Statistiques de l'Immigration, échapper à son attirail de trames serrées, ses filins, ses treuils, ses filets, ses tissus urbains, ses tuilages, ses toiles d'araignées, ses filigranes, ces contrôles d'identité, ses faciès, ses charters, trains de nuit et bateaux, oil de verre des caméras, plans Vigipirate, mouchards, recruteurs, flics, agents multiples chargés de vous socialiser ou de vous expulser. (p. 50)

Dans ce monde clos et réfractaire à toute présence d'autrui, est-il possible de résister, de s'ouvrir aux autres ? La réponse réside dans la radicalisation de sa condition qui exige de celui qui tente l'expérience de s'ouvrir un peu plus à son propre espace intérieur :

La solitude, ainsi que le refoulement de nombreux désirs sombres et dangereux, affirme D. Z., sont parmi les rares formes possibles de la liberté. (p. 34)

Devenu anonyme, parvenu à un degré de conscience supérieur, D. Z. radicalisera un peu plus sa position en se pliant à cette exigence fondamentale qui lui dicte de pousser plus loin son expérience et d'en faire une manière spécifique de se tenir dans la vérité :

J'aboutissais à une constatation d'ordre chirurgical : n'être lié à rien, n'avoir à se justifier de rien ni devant personne. Assumer l'errance. (ibid.)

Cette troublante vérité, à laquelle parvient D. Z., ne fait qu'illustrer le nihilisme ambiant qui gouverne le monde de cette fin $\mathrm{du} \mathrm{xx}^{\mathrm{e}}$ siècle. Toutes les formes esquissées dans le roman attestent de l'effacement de l'être, et le passage précédent fait écho à la célèbre formule : "Je n'existe pas, le fait est notoire ", d'un des personnages de Beckett. La fin du roman creuse davantage l'absurdité d'une existence sans relief et sans enthousiasme :

Dans les galeries et les couloirs de correspondance du métro et du RER, j'entraperçois quelquefois le visage de ces femmes et de ces hommes lancés, dès tôt le matin, sur l'autoroute du quotidien, au pas de course. Je me demande si je leur suis semblable, si moi aussi, $j$ 'habite les confins de ces absences dont ils portent l'entrelacs de leurs traits. (p. 202-203) 
Recherches \& Travaux $-\mathrm{n}^{\circ} 76$

\section{Je est / et un autre}

Le travail de soi sur soi prend la forme, dans le roman, même au plus profond de la crise, d'un véritable plaidoyer pour la quête de l'Autre. Tout se règle désormais dans le périmètre d'un foyer installé sur un non-territoire. Bien que localisé, cet espace est en fait un endroit doublement déterritorialisé : il héberge des étrangers, tout en étant lui-même étranger à l'espace auquel il se rattache :

Le foyer est un pays étranger, une zone franche qui n’appartient à aucune terre. Pays de personne où un reliquat de rêves résiduels s'obstine à mentir à la vie pour la maintenir dans une frêle possibilité. (p. 194)

L'étranger qui renonce à une identité factice et assume sa condition d' "errant" ne peut "habiter» à l'étranger qu'en territoire étrange(r). C'est cette ambiguïté existentielle qui fait en quelque sorte des «errants" des êtres à la fois étranges et étrangers. Aussi, cette condition favorise-t-elle à la fois la promiscuité et la proximité : toutes les histoires d'amitié qui se développent dans le roman ont pour cadre spatial le foyer. Étrangement, ce n'est pas l'opulence qui devrait garantir la richesse et faciliter l'intégration qui rapprochera les hommes, mais son contraire, la misère, qui deviendra de fait un élément fédérateur. Peut-être, le dénuement nous dispense-t-il de quelque fardeau difficile à porter. D. Z. peut alors vivre au-delà des lois des hommes, libre de tout souci, de toute histoire :

Je ne voulais plus être d'aucune histoire, confesse-t-il, les histoires des hommes sont si lourdes, leur poids si écrasant, qu'elles obligent quelquefois à vivre à genoux. (p. 84)

Ce choix extrême affectera également l'ensemble des personnages du roman. Sien choisit de vivre dans le rêve et dans un pays de vent :

Moi je suis une rêveuse, dit-elle, ce que j'aime dans la vie c'est rêver et rire ça fait du bien de rire et quand il faut rêver et qu'en même temps il faut arrêter de rêver pour vivre ça devient compliqué. (p. 84)

Rêve et paroles déstructurés d'une étrange étrangère qui occupe une place importante dans le texte, manifestation concrète du déracinement et de l'errance.

Vivre dans un non-lieu, un espace qui refuse tous les marquages, c'est refuser toutes les logiques géographiques que l'homme a inventées pour mieux se nier. À mesure que progressent les logiques d'enfermement à l'origine de la séparation, l'homme se fait plus lointain et donc moins humain. Et c'est pour cette raison, entre autres, que D. Z. refuse l'idée même de patrie, 
éventail brandi par ceux qui ont besoin de justifier l'existence de leur terre et partant de leur tare :

Le choix, encore informulé, d'être un apatride - apatride est également un mot imprécis, car il n'est pas débarrassé de l'idée de patrie, c'était de l'idée même que je voulais me dépouiller, de même que celui de la clandestinité, ne représentaient sûrement pas une révolte contre une quelconque condition de paria subie à mon corps défendant, n’avaient rien de révolutionnaire, ne visaient nullement au rétablissement d'une justice bafouée par un ordre écrasant. Ce n'était pas non plus un cri d'alarme adressé aux bonnes consciences des humanitaires, je le désirais pardelà toutes les logiques possibles et admises, ne se pliant qu'à la sienne propre. Car il en avait bien une contrairement à ce que l'on aurait pu penser. (p. 33)

L'exil maintient dans toute leur vigueur les questions fondatrices qui ne s'accommodent pas d'explications chiffrées. Il reste, en effet, alors que «tout était pensé, classé, rangé, archivé» (p. 9), à pousser plus loin, plus en avant ce qui reste encore à penser, bref, «à penser l'impensable» (ibid.). Ainsi peut-on parvenir à la question décisive qui implique une dimension jusque-là impensée et liée à cet agrandissement métaphysique, ainsi qu'il est dit dans ce passage :

Je ne voulais plus être d'aucune histoire, les histoires des hommes sont si lourdes, leur poids si écrasant, qu'elles obligent quelquefois à vivre à genoux. (p. 32)

\section{La folie et les autres}

C'est à la périphérie et dans l'anonymat des situations communes que D. Z. sent renaître douloureusement la conscience de sa singularité. Cette position lui permet d'accomplir un spectaculaire renversement métaphysique, en redéfinissant les sens admis qui fixent arbitrairement des normes :

La folie c'est la pensée des autres, les gens normaux. Je demeure un être social et donne à tous l'impression réconfortante de faire partie d'un monde où l'on assigne une place à chacun. Dans ma tête seulement je suis libre. Et c'est là que je suis marginal, et sauvage. C'est là que je suis seul. (p. 202)

C'est dans cette situation singulière, "proche de la dissolution» (p. 79), que D. Z. retrouve la force d'appellation intacte de la révélation qui lui ouvre le monde de l'impensé :

Les vérités les plus simples, dit-il, celles que nous passons nos vies à nous compliquer, nous n'arrivons pas à les atteindre par la pensée, elles nous sont révélées. (p. 74)

En redéployant la pensée dans les reliefs et les creux de ce qui échappe à la raison, D. Z. réussit à échapper aux dénégations et aux illusions 
inquiétantes provenant de savoirs positifs qui enveloppent tout dans une rationalité rassurante :

D. Z. ne portait en lui rien qui fût morbide, il désirait conjurer dans son regard ce qui meurt dans les hommes, et plus que leur mort physique, leur humanité terrassée par des puissances qu'ils ont eux-mêmes élevées au rang de divinité. (p. 198)

Cette ouverture sur le dehors des choses permet à D. Z. de rejoindre le lieu des êtres réels, le territoire de la fraternité où la folie a un sens et donne une capacité d'expérience et de vérité. Le foyer, "étrange pays étranger», se singularisant par sa capacité et sa vocation à réunir les "poitrinaires qui ne veulent plus rentrer chez eux", change de vocation et devient le lieu même où il est possible de fonder une patrie. C'est en effet, dans ce lieu de l'extrême, "pays de personne", qu'ont lieu les possibles rencontres de tous ceux qui sont "installés dans le regret d'un pays» (p. 137), et qui ont "déserté le monde des humains qui ne l'étaient plus» (p. 73). C. K., dans sa quête d'absolu et son désir d'ouverture au delà des limites que l'existence ordinaire resserre sur chaque chose, incarne le sens vrai d'une force centrifuge qui ne s'accommode pas d'un territoire délimité par la folie des hommes:

C. K. est venu dans ma chambre. Il m’a annoncé qu'il partait. Bientôt. Cuba, l'Équateur, la Bolivie, Buenos Aires, peut-être le Chili, jusqu'à la Patagonie. (p. I4I)

Il avait constamment besoin de partir C. K., à la recherche d'un sens à ce qu'il sentait se dérégler en lui, ou pour éprouver plus intensément l'inconfort de la vie réelle avant que celle-ci ne lui échappe. (p. I44)

S'ouvrir à l'illimité de l'espace comme le fait C. K. qui ne sait pas d'où il vient, c'est passer de l'autre côté de ce qui est donné à vivre pour gagner le "pays du vent", là où la folie s'impose raisonnablement comme une condition nécessaire de dépassement des contingences aliénantes. Sien, dans son innocence toute-puissante et ses maladresses toutes enfantines, croit connaître les origines de celui avec lequel elle partage sa folie, le seul ordre qui aide à tenir face au grand désordre du monde :

C. K. Il a dû venir de par là-bas là où il y a la mer et beaucoup de vent. Je lui ai pas demandé où il était son pays mais je suis sûre que c'est un pays du vent. (p. 86)

Le pays du vent, c'est l'espace intérieur de l'être qui se tient au cœur de la détermination du monde et révèle les choses dans leur essence et leur vérité première. Lêtre de vent est un être de la folie essentielle qui transcende la réalité d'une existence réglée sur l'avidité et la possession des choses de la terre. Vivre dans le vent comme le fait C. K., c'est renoncer au confort garanti 
par les privilèges d'un territoire et d'une vie stable et sans risque au profit d'une vérité ontologique qui réconcilie l'homme avec sa vocation initiale. La réflexion de Sien révèle toute la profondeur de ce que peut être la vraie nature de tous ceux qui refusent que leur destin soit lié inexorablement à la dure loi de la limite :

Les gens qui ont du vent dans la tête, dit Sien à propos de C. K., je me dis qu'ils ne peuvent pas en même temps y mettre de la méchanceté parce qu'ils ont plus de place pour ça. (p. 89)

Cette vérité saisissante s'applique à tous les personnages du roman dont le destin est semblable à celui de l'arbre qui croît dans les conditions extrêmes. La relation vent/arbre nous restitue dans toute sa force brutale, mais nécessaire, le tragique d'une existence soumise à la «sagesse» de la seule force capable de nous rendre notre propre force défaite ailleurs :

Dans le Sud de la France, là où pour endiguer la force du mistral, de la tramontane et du ponant on plante aussi des haies de cyprès, les peupliers poussent différents, ils sont étiques, décharnés, mais ostentatoires, et à l’automne, ils prennent des couleurs qui parent leur beauté d'un tragique qui vous saisit à la gorge. (p. 26-27)

L'un des éléments fondateurs de la "géographie éolienne» n'est autre que l'imaginaire, puissance fédératrice par excellence, où les lois de l'hospitalité puisent leur force et leur vérité. Du coup, les concepts qui fondent les échanges et les relations sont balayés par la force du vent, et le «territoire» qui sépare devient un lieu de proximité. Le foyer devient ainsi une métaphore et un opérateur de métamorphose où la singularité, le « tout autre" ne sont plus mesurés à l'aune de la loi castratrice de l'origine qui empêche toute affirmation, mais à la puissance du vouloir dire de chaque idiome, de chaque air musical, de chaque vent qui souffle :

Oui, un étranger est toujours un étranger pour l'autre, mais entre eux il y a le tout autre, le troisième terme, la relation qui les maintient dans leur singularité, qui est, d'une manière ou d'une autre, intraduisible’3.

\section{L'intraduisible lieu}

La perte du lieu n'est plus alors vécue comme un drame; ce serait, plutôt, le seul «événement» qui puisse fonder un nouveau rapport aussi bien avec soi qu'avec les autres. Ce véritable tournant révélera une autre modalité d'envisager

3. A. Khatibi, «Nationalisme et internationalisme littéraire», Visages de l'étranger, Denoël, I987, p. 174 . 
la problématique de l'exil. Le texte nous souffle alors l'idée d'un autre impératif : celui qui exige que l'on se dépouille des prothèses identitaires qui accentuent le marquage, et nous oblige plutôt à la mise à nu, équivalent symbolique de la mise à mort volontairement assumée :

J'étais libre, définitivement libre, et n'avais plus à me préoccuper de ce qui, bien ou mal, devait advenir. Je brûlais mes vaisseaux. Le passeport vert était tout ce qui me liait à mon pays, j'avais depuis longtemps rompu avec ce qui m'y restait comme famille et amis, gommé de ma mémoire adresses et numéros de téléphone. La carte de séjour m’apparaissait comme la matérialisation d'un mensonge violant l'essence de mon état d'apatride. Clandestin, c'était la seule manière d'être dans la tonalité juste de la réalité. Je n’avais fait que rétablir le mouvement de ma vie dans la vérité de sa trajectoire. (p. 32)

Soumis à l'humeur du vent, le "clandestin", n'ayant désormais plus d'attache, est délivré de toutes les pesanteurs psychologiques et morales. C'est dans l'abandon du signe totémique distinctif qui relie l'être à son groupe ou à sa tribu qu'il est possible de fonder une nouvelle relation débarrassée des signes encombrants et mutilants des «bonnes consciences des humanitaires» (p. 33). Ce qui est alors considéré comme un facteur qui rendrait l'éloignement inévitable devient ici un principe qui fonde une proximité agissante : brassage d'idiomes et de cultures; proximité des continents qui ne sont plus séparés par les océans d'incompréhension; amitié entre D. Z., C. K., Sien, les Arabes, les Turcs et les migrants d'Afrique noire; ceux que le vent, en tournant dans tous les sens, a déposés sur les frontières de paysages inconnus :

En allant chez les putains, j'ai reconnu chez certaines d'entre elles le sentiment d'une fraternité déchue et pathétique, le souvenir ancien de rires que j'avais quittés en désertant le monde des humains qui ne l'étaient plus. (p. 72-73)

La perspective est ainsi renversée : quand on perd son rire et que le paysage intérieur s'est dangereusement assombri, ce sont les personnages "désintégrés " et "ténébreux» qui aménagent réellement une voie d'accès au-dedans de l'être. D. Z. peut ainsi rejoindre tous ceux qui ont choisi, pour ne pas déserter leur humanité, le difficile chemin de l'exil :

Je voulais conserver intacte mon humanité, en dépit de ma sortie du rire, et cheminer en paix à la rencontre du silence, retrouver la juste hauteur de ma respiration, pour me reposer un peu. Quand on ne sait plus rire, il est préférable de se taire.

Il est des paroles que l'on dit, qui nous font passer pour des fous. Il vaut mieux se la fermer pour ne pas entendre l'écho malmené de ses mots dans la voix des autres. (p. 64)

Promu au silence de l'exil, comme celui qui enveloppe originairement les fonds sous-marins, D. Z. se laisse emporter vers le large, le lieu de sa vérité. 
Le titre, traduction d'une célèbre chanson du terroir, traduit une vérité ontologique et permet de vérifier une expérience qu'on voit à l'œuvre tout au long du texte. À la solitude sociale imposée par autrui, répond un espace métaphorique, mais aussi plus authentique, qui permet à $\mathrm{D}$. Z . de développer une stratégie de survie. La formule "Je fais comme fait le nageur dans la mer» consacre, dès l'entame, la "ruse» qui rythmera tout au long de la traversée la démarche de qui "évolue au-dessus du vide total, au-dessus du néant» (p. 202).

Le texte, en récupérant des fragments de la culture populaire et de textes poétiques du patrimoine culturel universel, renverse les conceptions fétichistes des origines. Nous ne pouvons atteindre les autres dans ce qu'ils ont d'irrémédiablement autre que si nous acceptons, au prix d'un incroyable effort intellectuel, de renoncer à ce qui alourdit notre être. Il faut être capable de prendre du recul pour être capable d'un engagement vrai, qui est toujours aussi un engagement dans la vérité. Les "post-it» qui strient le texte sont des éléments fédérateurs au premier degré d'une conscience universelle. Le roman est, à ce titre, celui de tous les exilés et des victimes de la dure loi de la dépossession. C'est dans l'intraduisible lieu qu'a lieu vraiment le séjour :

Ici, tout est re-version intérieure : non seulement tout reste tourné vers le véritable "dedans" de la conscience, mais encore à l'intérieur de ce "dedans», tout se déverse pour nous, sans aucune borne, l'un dans l'autre. [...]. Dans cette intériorité nous sommes libres, car nous sommes en dehors du rapport entretenu par les objets ob-jetés autour de nous, et qui ne nous abritent qu'apparemment ${ }^{4}$.

Le «monde interprété» ne nous assure, en fin de compte, qu'une sécurité toute relative : «Nous ne sommes sûrs que là où nous ne comptons ni avec le défaut d'abri, ni sur un abri érigé dans, et par le vouloirs." Aucun discours, hormis celui de la poésie, essentiellement extérieur au monde des objets et à celui des relations codifiées, ne peut en effet traduire l'intraduisible et l'ininterprétable lieu à partir duquel se dit l'être :

Sous les signes des grands besoins de la vie et de ses grandes contraintes, nous dit Yves Bonnefoy, la langue n'est pas vouée seulement à décrire les apparences, mais à nous tourner vers autrui pour fonder avec lui un lieu et décider de son sens ${ }^{6}$.

Le cheminement intellectuel de $\mathrm{D}$. Z . nous oblige ainsi à réfléchir, non pas sur les valeurs que nous élaborons pour réaliser ce qui nous paraît l'essentiel de la vie même, mais sur ce que, fondamentalement, nous n'avons pas à abriter en nous, "cette profondeur substantielle qui apaise le besoin d'être,

4. M. Heidegger, «Pourquoi les poètes ?», Chemins qui ne mènent nulle part, trad. W. Brokmeir, Gallimard, 1962, p. 37I.

5. Ibid., p. 358 .

6. Y. Bonnefoy, Entretiens sur la poésie, Neuchâtel, Éditions de la Baconnière, I98I, p. 2I. 
Recherches \& Travaux $-\mathrm{n}^{\circ} 76$

ou nous incite à nous ressaisir quand l'abstraction dans les mots a troublé l'approche de l'immédiat ${ }^{7} »$.

\section{Post-it}

Sien a depuis remis du henné sur ses mains

Bibo a grandi

C. K. a toujours du vent dans les yeux est vivant

7. Id., "Y a-t-il une vérité poétique ?», Vérité poétique et vérité scientifique, Presses universitaires de France, 1989, p. 48. 